\title{
BKL Method in the Bianchi IX Universe Model Revisited
}

\author{
Leandro Meléndez Lugo ${ }^{1} \&$ Pablo Ag. Chauvet ${ }^{2}$ \\ ${ }^{1}$ Instituto Nacional de Investigaciones Nucleares, Centro Nuclear de México, Departamento de Física, México D. \\ F., México \\ ${ }^{2}$ Universidad Autónoma Metropolitana-Iztapalapa,Departamento de Física, México D. F., México \\ Correspondence: Leandro Meléndez Lugo, I Instituto Nacional de Investigaciones Nucleares, Centro Nuclear de \\ México, Departamento de Física, México D. F. 52750, México. E-mail: leandro.melendez@inin.gob.mx
}

Received: September 3, 2013 Accepted: November 7, 2013 Online Published: November 21, 2013

doi:10.5539/apr.v5n6p107

URL: http://dx.doi.org/10.5539/apr.v5n6p107

\begin{abstract}
Given the extreme difficulty in finding analytical solutions to Einstein's equations for universe models, such as the Bianchi type IX, different physical-mathematical techniques have been designed to attempt to find, at least approximate solutions that could have relevance in modern cosmology. Belinskii, Khalatnikov and Lifshitz (BKL) have developed a method to study a particular time evolution dynamic of the Bianchi type IX universe model. Herein, it is shown that this method contains pitfalls. These pitfalls, among other things, prevent the conclusion that this model provides chaotic behaviour. We show that the initial presumption made by BKL turns out to be inconsistent with the assumed initial conditions, since it cannot be kept along with the time-extended BKL process. That is, the Kasner stipulations corresponding to $\tau \rightarrow \infty$, taken as initial conditions, are at odds with the initial assumption imposed on Einstein's field equations.
\end{abstract}

Keywords: Einstein's equations, cosmology, general relativity, chaos in general relativity

\section{Introduction}

Derived from the extreme difficulty in finding analytical solutions for the Bianchi type IX universe model, fraught with an important inherent problem related to the singularities of the metric tensor components (Andriopoulos, 2008; Barrow \& Tipler, 1979; Belinskii \& Khalatnikov, 1970a, 1971; Belinskii, Khalatnikov, \& Lifshitz, 1970b, 1980; Bogoyavlenskii, 1973; E. M. Lifshitz, I. M. Lifshitz, \& Khalatnikov, 1971; Hawking \& Ellis, 1968), much research activity has been carried out with the aim of finding at least approximate solutions. Many attempts in this direction have been focused on ascertaining special techniques to confront the respective Einstein field equations (Barrow, 1988; Belinskii \& Khalatnikov, 1969; Belinskii, Lifshitz, \& Khalatnikov, 1970, 1982; Berger, 1997; Ferraz \& Francisco, 1992; Khalatnikov, Lifshitz, Khanin, Shchur, \& Sinai, 1985; Lifshitz \& Khalatnikov, 1963; Ryan, 1971a, 1971b; Springael, Conte, \& Musette, 1998; Wright \& Moss, 1985).

The well-known fact that some very simple dynamic systems with a minimal number of degrees of freedom, no stochastic force and regular initial data may show unpredictable or random behaviour has led to the search for the chaotic conduct of homogeneous cosmological models in the context of general relativity (Barrow, 1981, 1982; Chernoff \& Barrow, 1983; Cornish \& Levin, 1997; Latifi, Musette, \& Conte, 1994; Lin \& Wald, 1990; Zardecki, 1983). Currently, the so called Mixmaster universe model - a particular Bianchi type universe (Barrow, 1981, 1982; Chernoff \& Barrow, 1983; Latifi, Musette, \& Conte, 1994; Lin \& Wald, 1990; Misner, 1969)—has brought into existence a great number of papers in the literature asserting its chaotic conduct (Barrow, 1981, 1982; Chernoff \& Barrow, 1983; Cornish \& Levin, 1997; Latifi, Musette, \& Conte, 1994; Lin \& Wald, 1990; Misner, 1969; Zardecki, 1983). Accordingly, the verdict has been that this deterministic model is unpredictable with respect to the number of Kasner epochs that comprise each major cycle of its time evolution (Balinskii \& Khalatnikov, 1970a; Misner, 1969). Physical systems governed by non-linear equations of motion are candidates to exhibit chaos, and certainly many examples from all disciplines in physics are now known. In this paper, the main source, the technique to show that the Bianchi type IX universe model is chaotic, is critically reviewed (Belinskii \& Khalatnikov, 1970a). As is well known, this Bianchi model has been taken as an example of the possibly outstanding solution to Einstein's equations, with chaotic properties that have played the prime role in the study of chaos and quantum gravity (Barrow, 1981; Belinskii, Gibbons, Page, \& Pope, 1978; Hartle \& Hawking, 1983; Misner, 1969a, 1969b) in the field of general relativity (Barrow, 1981, 1982; Belinskii, 1978; 
Chernoff \& Barrow, 1983; Cornish \& Levin, 1997; Hartle \& Hawking, 1983; Latifi, Musette, \& Conte, 1994; Lin \& Wald, 1990; Misner, 1969a, 1969b; Zardecki, 1983).

The purpose of this study, which challenges directly the validity of the statement regarding the possible chaotic behaviour of the Bianchi IX universe model (Cornish \& Levin, 1997), is also to clear up and, therefore, question the validity of the main hypothesis supporting the foundation of those papers that pretend to demonstrate the random behaviour of the model; again, based on the never questioned existence of Kasner epochs in the aforementioned universe model (Barrow, 1981, 1982; Cornish \& Levin, 1997; Chernoff \& Barrow, 1983; Latifi, Musette, \& Conte, 1994; Lin \& Wald, 1990; Zardecki, 1983).

Belinskii, Khalatnikov and Lifshitz (BKL) were among the first to develop a discrete method to study the Mixmaster dynamics (defined to describe the evolution of a spatially homogeneous vacuum and diagonal Bianchi type IX cosmology) (Belinskii \& Khalatnikov, 1969, 1970a, 1971; Belinskii, Khalatnikov, \& Lifshitz, 1970b; E. M. Lifshitz, I. M. Lifshitz, \& Khalatnikov, 1971; Lifshitz \& Khalatnikov, 1963). Since then, the Mixmaster universe model has been purported to represent Einstein's theory, which exhibits the chaotic dynamic behaviour of a Hamiltonian system. Furthermore, this model is also considered to reflect the properties of solutions to more general Einstein equations. Other authors have criticized the BKL work from another point of view (Barrow \& Tipler, 1979; Latifi, Musette, \& Conte, 1994); here, it is shown that this method contains pitfalls which warn about the conclusion that Kasner epochs exist in an approximate solution to the Bianchi IX universe with its alternating scale factors and chaotic behaviour.

\section{The BKL Technique}

In essence, BKL method attempts to find an approximate solution to Einstein's field equations in the Mixmaster universe model, a coupled system of differential equations written as: (Belinskii \& Khalatnikov, 1970a; Belinskii, Khalatnikov, \& Lifshitz, 1970b)

$$
\begin{gathered}
\frac{d\left(\frac{d a}{d t} b c\right) / d t}{a b c}+\left[a^{4}-\left(b^{2}-c^{2}\right)\right] /\left(2 a^{2} b^{2} c^{2}\right)=0 \\
\frac{d\left(\frac{d b}{d t} a c\right) / d t}{a b c}+\left[b^{4}-\left(c^{2}-a^{2}\right)\right] /\left(2 a^{2} b^{2} c^{2}\right)=0 \\
\frac{d\left(\frac{d c}{d t} a b\right) / d t}{a b c}+\left[c^{4}-\left(a^{2}-b^{2}\right)\right] /\left(2 a^{2} b^{2} c^{2}\right)=0 \\
\frac{d^{2} a / d t^{2}}{a}+\frac{d^{2} b / d t^{2}}{b}+\frac{d^{2} c / d t^{2}}{c}=0
\end{gathered}
$$

where $a, b$ and $c$ represent the universe scale factors.

BKL method, with the aim of rewriting Einstein's field equations in a more compact manner (in order to apply their above-mentioned method), defines the transformation:

$$
a=\exp (\alpha), \quad b=\exp (\beta), c=\exp (\gamma), d t=a b c d \tau
$$

By substituting this transformation into Equation (1), one obtains that:

$$
\begin{gathered}
2 \alpha_{\tau \tau}=(\exp (2 \beta)-\exp (2 \gamma))^{2}-\exp (4 \alpha) \\
2 \beta_{\tau \tau}=(\exp (2 \gamma)-\exp (2 \alpha))^{2}-\exp (4 \beta) \\
2 \gamma_{\tau \tau}=(\exp (2 \alpha)-\exp (2 \beta))^{2}-\exp (4 \gamma) \\
(\alpha+\beta+\gamma)_{\tau \tau} / 2=\alpha_{\tau} \beta_{\tau}+\alpha_{\tau} \gamma_{\tau}+\beta_{\tau} \gamma_{\tau}
\end{gathered}
$$

Adding Equations (3) and substituting the result into Equation (4) the second order derivatives are eliminated to obtain the first integral of the system of Equations (3):

$$
\begin{gathered}
\alpha_{\tau} \beta_{\tau}+\alpha_{\tau} \gamma_{\tau}+\beta_{\tau} \gamma_{\tau}=[\exp (4 \alpha)+\exp (4 \beta)+\exp (4 \gamma) \\
-2 \exp (2 \alpha) \exp (2 \beta)-2 \exp (2 \alpha) \exp (2 \gamma)-2 \exp (2 \beta) \exp (2 \gamma)] / 4
\end{gathered}
$$

Before proceeding with this development, BKL method makes a deviation to treat a more elemental universe 
model. A very simplified problem (Bianchi type I) results when the squared parentheses of Equation (1) are set equal to zero, then we obtain the Kasner solution:

$$
\alpha \sim \ln t^{P_{1}}, \quad \beta \sim \ln t^{P_{2}}, \quad \gamma \sim \ln t^{P_{3}}
$$

That is

$$
a \sim t^{P_{1}}, \quad b \sim t^{P_{2}}, c \sim t^{P_{3}}
$$

with

$$
a b c=\Lambda t, \tau=\ln t / \Lambda+c o n s t
$$

where $\Lambda$ is a constant.

Here, $P_{1}, P_{2}, P_{3}$ are three arbitrary numbers satisfying the relation

$$
P_{1}+P_{2}+P_{3}=P_{1}^{2}+P_{2}^{2}+P_{3}^{2}=1
$$

Therefore, only one of these numbers is independent. They are never equal and are equal in pairs only in the two following cases: $(-1 / 3,2 / 3,2 / 3)$ and $(0,0,1)$. In all other cases they are distinct, one of them being negative and two positive. We shall order them by

$$
P_{1}<P_{2}<P_{3}
$$

Then they lie in the intervals

$$
-1 / 3 \leq P_{1} \leq 0, \quad 0 \leq P_{2} \leq 2 / 3,2 / 3 \leq P_{3} \leq 1
$$

The numbers $P_{1}, P_{2}, P_{3}$ can be represented in parametric form:

$$
\begin{gathered}
P_{1}(u)=-u /\left(1+u+u^{2}\right) \\
P_{2}(u)=(1+u) /\left(1+u+u^{2}\right) \\
P_{3}(u)=u(1+u) /\left(1+u+u^{2}\right)
\end{gathered}
$$

As the $u$ parameter varies in the range $u \geq 1, P_{1}, P_{2}$ and $P_{3}$ assume any of their possible values preserving the order defined in (8). The values $u<1$ lead to the same range of values of $P_{1}, P_{2}, P_{3}$ since

$$
P_{1}(1 / u)=P_{1}(u), \quad P_{2}(1 / u)=P_{3}(u), P_{3}(1 / u)=P_{2}(u)
$$

After this, BKL method affirms that, at a certain moment of the Mixmaster time evolution, all terms on the right hand side of Equations (3) can be ignored and then we have a Kasner solution. However, such a situation cannot persist, since there are always such terms on the right hand side of Equations (3) which are increasing. So, when the right hand side of Equations (3) is ignored the solutions are Equations (7).

In the next step, BKL method considers that the Kasner system is perturbed. Suppose that the perturbation is the term $a^{4}$ which is then dominant; and $b, c$ terms are ignored preserving only $a^{4}$ on the right hand side of Equations (3). Then, the perturbed Kasner system is

$$
\begin{aligned}
& \alpha_{\tau \tau}=-\exp (4 \alpha) / 2 \\
& \beta_{\tau \tau}=\gamma_{\tau \tau}=\exp (4 \alpha) / 2
\end{aligned}
$$

A Kasner epoch is defined as that part of the time evolution of the general solution to the problem of Kasner Bianchi type I, specified by the values of four parameters, in the solution of another universe model such as the Bianchi IX, where the product of the three scale factors is proportional to the cosmological time $(a b c=\Lambda t)$.

The solutions of Equations (11) describe the time evolution of the simplified Einstein equations, from their initial state (Kasner epoch). The initial conditions are as follows

$$
\tau \rightarrow \infty, \alpha_{\tau}=\Lambda P_{1}, \quad \beta_{\tau}=\Lambda P_{2}, \gamma_{\tau}=\Lambda P_{3}
$$

With these initial conditions, BKL method considers the evolution of the model in the time direction $t \rightarrow 0$, therefore, the initial conditions correspond to a later rather than an earlier moment of time.

Equations (11) are then integrated. The solution satisfying the conditions in Equation (12) has the form:

$$
a^{2}=2 \Lambda\left|P_{1}\right| \sec h\left(2 \Lambda\left|P_{1}\right| \tau\right)
$$




$$
\begin{aligned}
& b^{2}=b_{0}^{2} \exp \left(2 \Lambda\left(P_{2}-\left|P_{1}\right|\right) \tau\right) \cosh \left(2 \Lambda\left|P_{1}\right| \tau\right) \\
& c^{2}=c_{0}^{2} \exp \left(2 \Lambda\left(P_{3}-\left|P_{1}\right|\right) \tau\right) \cosh \left(2 \Lambda\left|P_{1}\right| \tau\right)
\end{aligned}
$$

where $b_{0}, c_{0}$ are constants.

It is easy to verify that in the limit $\tau \rightarrow \infty$ the asymptotic form of the functions in Equation (13) is identical with the power laws in Equation (7). In the limit $\tau \rightarrow-\infty$, the asymptotic form of these functions, and that of the function $t(\tau)$, are given by

$$
\begin{gathered}
a \sim \exp \left(-\Lambda P_{1} \tau\right) \\
b \sim \exp \left(\Lambda\left(P_{2}+2 P_{1}\right) \tau\right) \\
c \sim \exp \left(\Lambda\left(P_{3}+2 P_{1}\right) \tau\right) \\
t \sim \exp \left(\Lambda\left(1+2 P_{1}\right) \tau\right)
\end{gathered}
$$

Expressing $a, b$ and $c$ as a function of $t$, BKL method obtains

$$
a \sim t^{P_{1}^{\prime}}, \quad b \sim t^{P_{2}{ }^{\prime}}, c \sim t^{P_{3}}
$$

where

$$
\begin{gathered}
P_{1}^{\prime}=\left|P_{1}\right| /\left(1-2\left|P_{1}\right|\right) \\
P_{2}^{\prime}=\left(2\left|P_{1}\right|-P_{2}\right) /\left(1-2\left|P_{1}\right|\right) \\
P_{3}^{\prime}=\left(P_{3-} 2\left|P_{1}\right|\right) /\left(1-2\left|P_{1}\right|\right)
\end{gathered}
$$

and

$$
a b c=\Lambda^{\prime} \tau, \quad \Lambda^{\prime}=\left(1-2\left|P_{1}\right|\right) \Lambda
$$

After this result, BKL method affirms that the effect of the perturbation is to replace one Kasner epoch by another, where the negative power of $t$ is transferred from the scale factor $a$ to $b$, that is, if originally $P_{1}$ is negative, then in the new solution we find $P_{1}<0$.

In short, the whole BKL process begins at a Kasner epoch $(\tau \rightarrow \infty)$, and later arrives at an intermediate (perturbed) stage described by solution (13). Then, taking the limit $\tau \rightarrow-\infty$ (the asymptotic form for these functions) one arrives at another, different, Kasner epoch. In fact, in the next Kasner epoch, the $u$ parameter assumes the value $u$, $=u-1$ and the constant $\Lambda$ is replaced by $\Lambda^{\prime}=\left(1-2\left|P_{1}(u)\right|\right) \Lambda$. This result is equivalent to the process of changing the $u$ parameter by $u-1$ in Equations (10), to obtain the new $P^{\prime}$ values given in Equations (16).

At the end of their third section, referring to the $a$ and $b$ scale factors BKL method states: if the absolute values of these terms are close to each other at the beginning of a long era-the moment when one Kasner epoch replaces another - they remain close during a long part of the duration of the whole era. In this case, which they call the case of small oscillations, it is insufficient to consider only one type of perturbation. It is then necessary to take into account, simultaneously, the effect of two perturbations.

BKL method begins the fourth section analysing the case of small oscillations, where it is considered that $a$ and $b$ are small, and oscillate so that their absolute values remain close to each other while the third scale factor $c$ decreases monotonically. Since the $c$ function decreases quickly, they investigate the solution of Equations (3) in the region in which $c$ can be ignored compared to $a$ and $b$.

Ignoring the function $c$, they write the first two Equations (3) in the form:

$$
\begin{gathered}
\alpha_{\tau \tau}+\beta_{\tau \tau}=0 \\
\alpha_{\tau \tau}-\beta_{\tau \tau}=\exp (4 \beta)-\exp (4 \alpha)
\end{gathered}
$$

while the third is (see Equation (5))

$$
\gamma_{\tau}\left(\alpha_{\tau}+\beta_{\tau}\right)=-\alpha_{\tau} \beta_{\tau}+(\exp (2 \alpha)-\exp (2 \beta))^{2} / 4
$$

In this fourth section, BKL method proceeds in a different manner from that the third where, beginning in a Kasner epoch, they later proceed to a perturbed stage, and then from Equations (13) take the limit as $\tau \rightarrow-\infty$ (the asymptotic form of these functions) arriving at another Kasner epoch. The transition from an initial Kasner 
epoch to the next is carried out by substituting Equations (10) in the parametric expression for the $P$ 's and substituting $u$ parameter by $u-1$. Here the mathematical steps followed are different from those used before. BKL method proposes a change in the independent variable $\tau \rightarrow \xi$, where

$$
\xi=\xi_{0} \exp \left(2 a_{0}^{2}\left(\tau-\tau_{0}\right) / \xi_{0}\right)
$$

Now, with $X=\alpha-\beta$, they obtain from (18)

$$
X_{\xi \xi}+X / \xi+\sinh (2 X) / 2=0
$$

and from (19)

$$
\gamma_{\xi}=-1 /(4 \xi)+3\left(2 X_{\xi}^{2}+\cosh (2 X-1)\right) / 8
$$

With the variable change (20) they obtain

$$
\begin{gathered}
a=a_{0} \sqrt{\xi} / \xi_{0}\left(1+A \sin \left(\xi-\xi_{0}\right) / \sqrt{\xi}\right) \\
b=a_{0} \sqrt{\xi} / \xi_{0}\left(1-A \sin \left(\xi-\xi_{0}\right) / \sqrt{\xi}\right) \\
c=c_{0} \exp \left(-A^{2}\left(\xi_{0}-\xi\right)\right)
\end{gathered}
$$

BKL method then discusses the region $\xi<<1$. The principal terms in the solution of Equation (21) are given by

$$
X=\alpha-\beta=k \ln \xi+q
$$

where $k$ and $q$ are constants, $k$ satisfying $-1<k<1$. This condition guarantees that the last term in Equation (21) is small $\left[\sinh (2 X)\right.$ contains $\xi^{2 k}$ and $\left.\xi^{-2 k}\right]$. Expressing $a, b, c$ and $t$ from the above equations, we obtain

$$
a \sim \xi^{(1+k) / 2}, \quad b \sim \xi^{(1-k) / 2}, \quad c \sim \xi^{-\left(1-k^{2}\right) / 4}, \quad t \sim \xi^{\left(3+k^{2}\right) / 4}
$$

This is again a Kasner solution, where the negative power of $t$ corresponds to $c(t)$. BKL method's section 5 begins with the phrase: "The eras of small oscillations discussed in section 4 violate the 'regular' evolution as defined in third section; this fact makes the investigation of the evolution more difficult over periods of time comprising many eras. However, it can be shown that in the asymptotic region of arbitrary small time periods $t$ (sufficiently far from the initial moment of time when arbitrary initial conditions are imposed) such 'anomalous' behaviour in the evolution of the model towards a singular point does not occur. Even for long eras, at the moment when one Kasner epoch replaces another, the two oscillating functions are so different that the actual replacement of one epoch by another is caused only by one type of perturbation".

After that, BKL method state that: In every Kasner epoch we have

$$
a b c=\Lambda t
$$

that is

$$
\alpha+\beta+\gamma=\ln \Lambda+\ln t
$$

As a result of the transition from one Kasner epoch to another, the constant $\ln \Lambda$ changes by an amount of order of 1 . In the asymptotic region when $|\ln t|$ is arbitrarily large, we can ignore not only such changes but also the whole constant $\ln \Lambda$. In other words, in this limit we ignore all quantities whose ratios to $|\ln t|$ tend to zero as $t$ $\rightarrow 0$.

Then we find

$$
\alpha+\beta+\gamma=-\Omega
$$

where $\Omega$ denotes the logarithmic time

$$
\Omega=-\ln t
$$

In this approximation, the interchange of two epochs is instantaneous. We can also ignore the constant $\alpha_{\max }=\ln \left(2\left|P_{1}\right| \Lambda\right) / 2$ on the right hand side of the equation $a_{\max }=\sqrt{2 \Lambda}\left|P_{1}\right|$, which defines the moment when one epoch replaces another, that is, we can replace this equation by the condition $\alpha=0$ (or alternatively, by the conditions $\beta=0$ or $\gamma=0$ if the initial negative exponent corresponds either to the function $b$ or $c$ ). Thus we assume that

$$
\alpha_{\max }=\beta_{\max }=\gamma_{\max }=0
$$

so that, quantities $\alpha, \beta, \gamma$ assume only negative values which are related to each other at every moment of time by 
expression (25).

Assuming the instantaneous interchange of two epochs, we neglect the "widths" of the transition regions compared to the duration of the epochs themselves; in fact, this is justified as will be shown later. The conditions $a_{\text {max }}=\sqrt{2 \Lambda\left|P_{1}\right|}$ can be replaced by condition (27) provided the quantity $\ln \left(\left|P_{1}\right| \Lambda\right)$ is small in comparison with the amplitudes of oscillations of the corresponding functions $\alpha, \beta, \gamma$. However, as already noted in the third section, during the transition from one era to another, very small values of $\left|P_{1}\right|$ can occur. Their magnitude and the probability that they occur are not related to the amplitude of the oscillations attained at that moment. Therefore, in principle $\left|P_{1}\right|$ can become so small that the above condition is not satisfied. Such a large decrease in $\alpha_{\max }$ can result in special situations when the matching of two Kasner epochs, defined by Equations (10), becomes incorrect (this includes the situation discussed in the fourth section). Such a dangerous situation would invalidate the arguments used in our statistical analysis given in the sixth section. However, as already noted, the probability of such cases tends asymptotically to zero.

Let us consider an era containing $k$ Kasner epochs, which correspond to the following values of

$$
u_{n}=k+X-1-n, n=0,1, \ldots . k-1
$$

Furthermore, let $\alpha$ and $\beta$ be the oscillating functions in this era.

Let us denote the beginning of an epoch characterized by the parameter $u_{n}$ by $\Omega_{n}$. At each moment $\Omega_{n}$, one of the quantities $\alpha$ and $\beta$ vanishes and the other reaches a minimum. We shall denote these minimum values of $\alpha$ or $\beta$ by

$$
\alpha_{n}=-\delta_{n} \Omega_{n}
$$

From the above considerations they obtain the approximate solution depicted in Figure 1, where the vertical strips represent the Kasner epochs, each having different temporal durations, and the small arrows in each one of the three functions $(\alpha, \beta$ and $\gamma)$ mark the time direction.

$\mathrm{Up}$ to now the main idea of the BKL technique, that describes the $a, b$ and $c$ asymptotic approach to the singularity $(t=0)$, has been presented.

The steps constituting the BKL method can be summarized as follows:

- Introducing the assumption that one of the three scale factors is much higher than the other two, on the model of universe Bianchi IX, a reduced equation system is obtained which preserves only the largest scale factor, in the curvature terms which is considered as a perturbation .

- $\quad$ This reduced system is solved. A Kasner type solution is imposed, as initial condition, to the reduced system solution obtained.

- In the limit of the logarithmic time $\tau \rightarrow-\infty$ the asymptotic form is obtained from this solution. In this asymptotic form the logarithmic time is replaced from $a b c=\Lambda t$ and $\Lambda \tau=\ln t / \Lambda+$ const, instead of $d t=a b c d \tau$. From here you get another Kasner.epoch. We conclude that the effect of the perturbation is replace a Kasner epoch for another with different $p$ parameters.

- $\quad$ Once the Kasner epochs are defined, the general problem is solved now with two perturbations during a long era with two scale factors oscillate so that their absolute values remain close to each other, say $a$ and $b$ and the third scale factor $c$ decreases monotonically.

- $\quad$ As the final part, the approximate solution to the Bianchi type IX is obtained by this method containing $k$ Kasner epochs, two scale factor oscillate and the third decreases monotonically. 


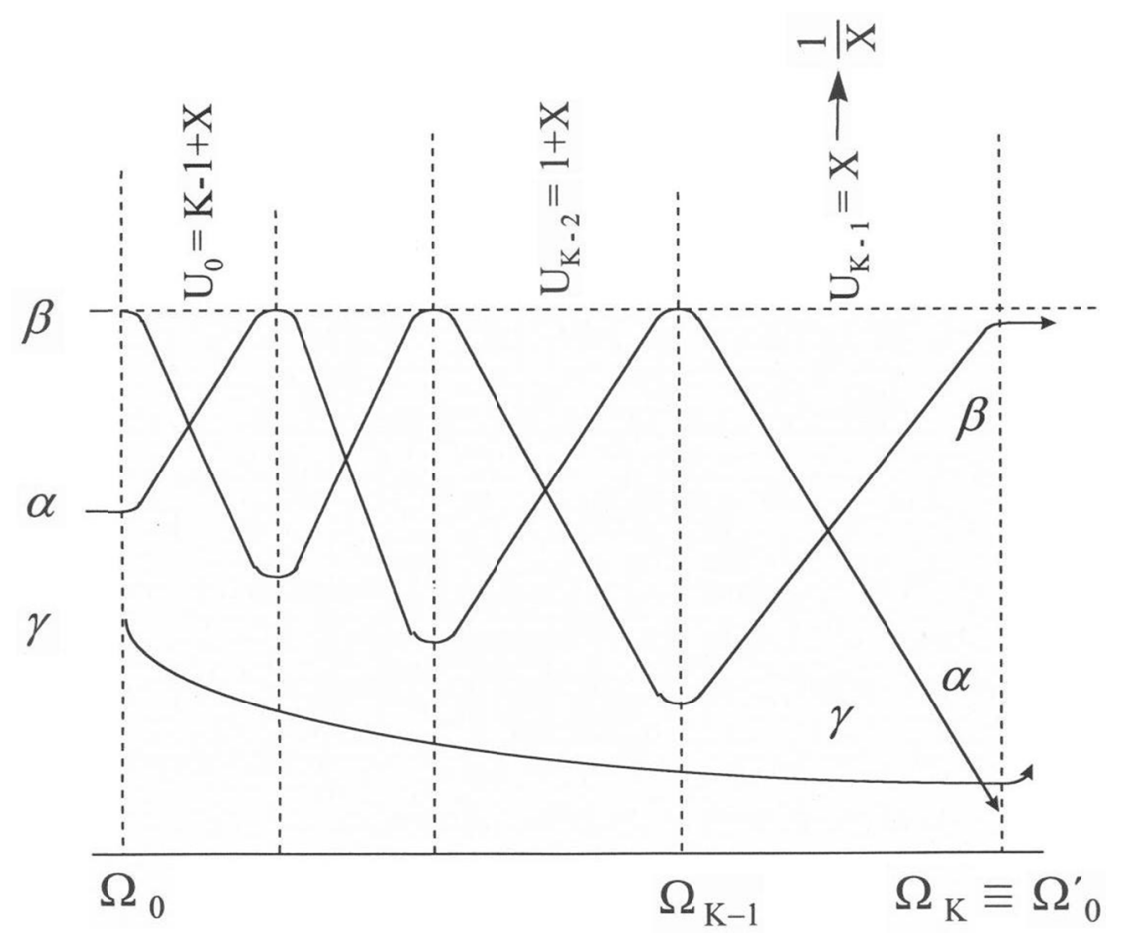

Figure 1. Graphic representation of the BKL method approximate oscillatory solution. An era containing $K$ Kasner epochs is shown, corresponding to the following values of the $u$ parameter. $u_{n}=K+X-1-n$,

$$
n=0,1, \ldots K-1
$$

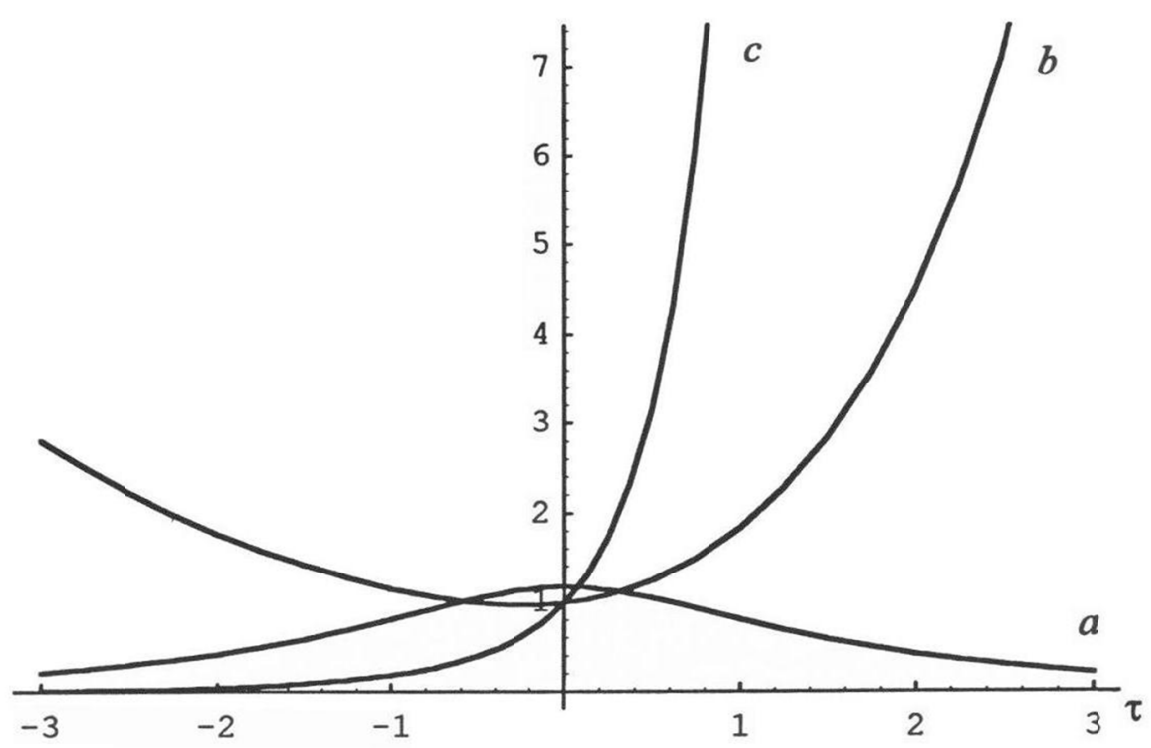

Figure 2. Graphic representation of the scale factors for the reduced Einstein's field equations in the BKL technique. Note that the $a$ function is smaller than the $b$ and $c$ functions (scale factors) in a vast part of the $\tau$ interval. This contradicts the initial assumption $a>b, c$

\section{Comments on BKL Inconsistencies}

1) Einstein's field equations (Equations (1)) describe the dynamics of a vacuum Bianchi type IX model. BKL first argue that one can always find a moment where the three curvature terms can be ignored thereby obtaining Kasner equations whose solutions are then taken as initial conditions to a perturbation problem: the Kasner equations are perturbed by assuming that, say $a>b, c$, that is, in the first approximation $b$ and $c$ are ignored 
subsequently to obtain Equations (11), after making a variable change given by Equations (2). However, as such, the system Equations (11) do not hold any indication that they come from a more complex system, Einstein's field equations (Equations (1)), where $b$ and $c$ are present. The solutions, Equations (13) to the system of Equations (11) when Equations (12) are taken as initial conditions, are depicted in Figure 2. From it, we see that the condition $a>b, c$ is rather untenable because actually $a<<b, c$ is an extended part of the $\tau$ interval.

2) The contradiction mentioned in Point 1 comes in part from imposing incongruous initial conditions as given by Equations (12), to reduced Einstein's field Equations (11). As $\tau \rightarrow \infty$, the negative $P_{1}$ parameter, which is smaller than the $P_{2}, P_{3}$ Kasner parameters (see Equation (9)) was assigned to $a$, and makes it smaller than the $b$ or $c$ scale factors, as given by Equation (6) over an extended part of the $\tau$ interval (Equation (2)) contradicting the initial assumption that $a \gg>b, c$. To circumvent this contradiction, one can attempt to formulate the initial conditions in a different manner but still one cannot obtain the initial assumption $(a>b, c)$. If, in order to have $a>>b, c$, one assigns $P_{3}$ to $a$, because $P_{3}>P_{2}>P_{1}$, as given by Equation (9), that is:

$$
\tau \rightarrow \infty, \alpha_{\tau}=\Lambda P_{3}, \quad \beta_{\tau}=\Lambda P_{2}, \gamma_{\tau}=\Lambda P_{1}
$$

then, the function $a$, whose expression is given in Equations (13), would now be written

$$
a^{2}=a_{0} \sec h a_{0} \tau
$$

with $a_{0}>0$. From Equation (2)

$$
\begin{gathered}
\alpha=\ln \left(a_{0} \sec h a_{0} \tau\right) / 2 \\
\alpha_{\tau}=-a_{0}\left(\tanh a_{0} \tau\right) / 2
\end{gathered}
$$

then as $\tau \rightarrow \infty$

$$
\alpha_{\tau}=-a_{0} / 2=\Lambda P_{3},
$$

Note that $a_{0}<0$

So, an imaginary function is obtained since, from Equation (29)

$$
a^{2}=-2 \Lambda P_{3} \sec h\left(-2 \Lambda P_{3}(\tau)\right)
$$

By the following considerations, it is possible to avoid the negative sign in Equation (31). Like in the two last Equations (13), Equation (29) can be written as

$$
a^{2}=a_{1}^{2} \sec h a_{0} \tau
$$

With $a_{1}{ }^{2}$ instead of $a_{0}$, in these terms from Equation (2), with $a^{4}=\exp (4 \alpha)$ we have

$$
\alpha_{\tau \tau}=-\exp (4 \alpha) a_{0}^{2} /\left(2 a_{1}^{4}\right)
$$

Then, by choosing the negative sign, Equation (32) can be written as

$$
a^{2}=2 \Lambda P_{3} \sec h\left(-2 \Lambda P_{3}(\tau)\right)
$$

But from (33) in the limit as $\tau \rightarrow \infty$ one obtains

$$
\alpha_{\tau}=-\Lambda P_{3}
$$

Then, Equation (34) contradicts the initial condition given by Equations (12).

Accepting the assumption that, for the solution of the reduced system (equations (11)) $a>>b, c$ is contrary to imposing the initial conditions given by Equations (28) or (12) it can be concluded that, initial conditions in agreement with the initial assumption $a>>b, c$ are impossible to find, since clearly from Equations (13) and Figure $2 b, c>a$ in most parts of the $\tau$ or $t$ interval.

3) In order to find the solution to the group of Equations (1), in the technique of BKL, described in their third section, the singularity consists of the steps beginning with a Kasner solution as $\tau \rightarrow \infty$ and continues with an intermediate perturbed stage represented by Equations (11) - the reduced system. Next, these solutions taken to the limit, as $\tau \rightarrow-\infty$, are asymptotic functions that represent a Kasner type solution with different values for the former $P_{1}, P_{2}$ and $P_{3}$ in the form of new parameters $P_{1}^{\prime}, P_{2}$, and $P_{3}{ }^{\prime}$. They argue that these new values, for example $P_{1}^{\prime}$ are generated because equation $\alpha_{\tau \tau}=-\exp (4 \alpha) / 2$ represents well the movement of a particle in the field of an exponential potential in one dimension with coordinate $\alpha$. When the particle is reflected from the wall of the well after colliding with it, this reflection produces a new value for $P_{1}$. Nevertheless, the change in 
value for these parameters can have another explanation. The BKL process, interpreted as a reflection, is achieved upon taking the limit as $\tau \rightarrow \infty$ on the one hand and then as $\tau \rightarrow-\infty$ on the other. From Figure 2, it can be seen that the slope of the function $a$ as $\tau \rightarrow \infty$ has a negative sign, while as $\tau \rightarrow-\infty$ it has a positive sign (a well-known property of the function $\sec h$ ). Then, upon taking the asymptotic forms of this function as $\tau \rightarrow \infty$ and $\tau \rightarrow-\infty$, an evident change of sign is due in the parameter $P_{1}$ in each extreme point. One can say the same thing for the scale factor $b$, as is easily seen in the same Figure 2 where its slopes at the afore mentioned ends have different signs. In other words, $P_{1}$ and $P_{2}$ must interchange their signs in the extremes of the $\tau$ interval. The function $c$ is monotonic and at the ends $\tau \rightarrow \infty$ and $\tau \rightarrow-\infty$ maintain its positive sign. Herein, the commentary is that, the statement of BKL that this pattern follows as a consequence of the exchange of Kasner epochs is very arbitrary without connection to the original Einstein's field equations.

4) BKL method begins the fourth section considering a long era during which they assume $a-b>>c$, calling it the case of small oscillations. With this assumption $(a, b>>c)$, from the Bianchi type IX Equations (3), BKL first obtain Equations (18) and (19) and its solutions (23) in terms of $\xi$ (see Equation (20)), for $\xi$ is relatively large, since these equations are obtained to first order in $1 / \xi$. However, when the values of the $\xi$ variable (as $t \rightarrow 0$ ) are such that $\xi<<1$ one finds that the solutions given in Equations (24), which themselves include the function $c$, have $c \gg>a, b$ contradicting the initial assumption that $a, b>>c$.

5) The aforementioned Kasner epochs in each one of the above cases, are joined through unknown but invented continuous functions among themselves connecting similar expressions. The result of this process is a succession of Kasner epochs that constitute the base to obtain the final approximate solution that has the oscillatory conduct represented in Figure 1. We remark that, due to the primary assumption $a>>b, c$ not being satisfied by the solutions obtained to Equations (11); the initial conditions, Equations (12) and (28), strongly oppose it, as already mentioned in point 1 . Therefore, the conclusion that the curves depicted in Figure 1 represent a possible approximate solution to the Bianchi IX model Equations (3) is unwarranted.

6) Explicitly according to the BKL technique, the change in the Kasner parameters $P_{1}, P_{2}$ and $P_{3}$ to new parameters $P_{1}{ }^{\prime}, P_{2}$ ' and $P_{3}$, signifies the existence of two contiguous Kasner epochs separated by an infinite $\tau$ interval, since $P_{1}, P_{2}$ and $P_{3}$ correspond to the limit $\tau \rightarrow \infty$ and $P_{1}{ }^{\prime}, P_{2}{ }^{\prime}, P_{3}$ ' to the limit $\tau \rightarrow-\infty$. Yet, the Kasner epoch corresponding to $\tau \rightarrow \infty$ taken as an initial condition is at odds, as discussed in point 2 with the primary assumption $a>>b, c$ imposed on the Bianchi IX Equations (3). So, the oscillatory behaviour of the solutions in Figure 1 is again unjustified.

\section{Concluding Remarks}

The BKL method approximate solution to Einstein's equations in the Bianchi type IX universe model, represented partially by Equations (13), does not satisfy the initial assumption $a>>b, c$ imposed on Equations (1) because of the Kasner type solutions assigned as initial conditions for the solutions of the reduced system Equations (11). They obtain $a<<b, c$ for an extended part of the $\tau$ interval. In other words, this initial condition is inconsistent with the initial assumption $a>>b, c$, leading to a contradiction $(a<b, c)$.

Moreover, BKL method states that they also mix two perturbations to construct the approximate solution of Equations (1) near the singularity. Both perturbations, that corresponding to the Kasner problem and that to the small oscillations case, do not satisfy their respective initial assumptions, that is, $a>>b, c$ in the Kasner case, and $a, b>c$ in the small oscillations case. This procedure leads to the now doubtful oscillatory solution depicted in Figure 1.

Explicitly, the asymptotic form of the solutions given by Equations (13) as $\tau \rightarrow \infty$ and $\tau \rightarrow-\infty$ is the mechanism through which the transformation of the parameter $u$ into $u-1$ is achieved. One can assert that the Kasner stipulations corresponding to $\tau \rightarrow \infty$ taken as the initial condition are at odds with the initial assumption $a>>b, c$ imposed on Bianchi type IX equations. Therefore, the course taken by BKL method to generate the oscillations that produce the different Kasner epochs of Figure 1 is unsound.

Finally, when a reduced system of differential equations was obtained, we lost all connection with the original system. In these conditions, it is likely that the solution found could be ingenious but rather far-fetched. The approximate solution in Figure 1 is interesting but lacking in links with the real Einstein's field equations.

\section{Acknowledgments}

We would like to thank CONACyT México Sistema Nacional de Investigadores (SNI) Grant No. 10793.

\section{References}

Andriopoulos, K., \& Leach, P. J. L. (2008). The Mixmaster Universe: the final reckoning? J. Phys. A: Math Theor, 41, 1-11. http://dx.doi.org/10.1088/1751-8113/41/15/155201 
Barrow, J. D., \& Tipler, F. J. (1979). Analysis of the Generic Singularity Studies by Belinskii, Khalatnikov, \& Lifshitz. Phys. Rep. C, 56, 371-402. http://dx.doi.org/10.1016/0370-1573(79)90097-8

Barrow, J. D. (1981). Chaos in the Einstein Equations. Phys. Rev. Lett., 46(15), 963-966. http://dx.doi.org/10.1103/PhysRevLett.46.963

Barrow, J. D. (1982). Chaotic Behaviour in General Relativity. Phys. Reports (Rev. Sect. Of Phys. Lett.), 85(1), $1-49$.

Barrow, J. D. (1988). The Premature Recollapse Problem in Closed Inflationary Universe. Nucl. Phys. B, 296, 697-709. http://dx.doi.org/10.1016/0550-3213(88)90040-5

Belinskii, V. A., \& Khalatnikov, I. M. (1969). On the nature of the singularities in the general solution of the gravitational equations. Sov. Phys. J. E. T. P., 29(5), 911-917.

Belinskii, V. A., \& Khalatnikov, I. M. (1970a). General solution of the gravitational equations with a physical singularity. Sov. Phys. J. E. T. P., 30(6), 1174-1180.

Belinskii, V. A., Khalatnikov, I. M., \& Lifshitz, E. M. (1970b). Oscillatory approach to a singularity point in the Relativistic Cosmology. Adv. Phys., 19, 525-573. http://dx.doi.org/10.1080/00018737000101171

Belinskii, V. A., \& Khalatnikov, I. M. (1971). General solution of the gravitational equations with a physical oscillatory singularity. Sov. Phys. J. E. T. P., 32, 169-172.

Belinskii, V. A., Gibbons, G. W., Page, D. N., \& Pope, C. N. (1978). Asymptotically Euclidean Bianchi IX metrics in Quantum gravity. Phys. Lett. B, 76(4), 433-435. http://dx.doi.org/10.1016/0370-2693(78)90899-7

Belinskii, V. A., Khalatnikov, I. M., \& Lifshitz, E. M. (1980). On the problem of the singularityies in the general cosmological solution of the Einstein equations. Phys. Lett., 77A(4), 214-216.

Belinskii, V. A., Lifshitz, E. M., \& Khalatnikov, I. M. (1982). A general solution of the Einstein equations with a time singularity. Adv. Phys., 31(6), 639-667. http://dx.doi.org/10.1080/00018738200101428

Berger, B. K., Garfinkle, D., \& Strasser, E. (1997). New algorithm for Mixmaster Dynamics. Class. Quantum Grav., 14, L29-L36. http://dx.doi.org/10.1088/0264-9381/14/2/001

Bogoyavlenskii, O. I., \& Novikov, S. P. (1973). Singularities of the cosmological model of the Bianchi IX type according to the qualitative theory of differential equations. Sov. Phys. J. E. T. P., 37, 747-755.

Chernoff, D. F., \& Barrow, J. D. (1983). Chaos in the Mixmaster universe. Phys. Rev. Lett., 50(2), 134-137. http://dx.doi.org/10.1103/PhysRevLett.50.134

Cornish, N. J., \& Levin, J. J. (1997). The Mixmaster universe is chaotic. Phys. Rev. Lett., 78, 998-1001. http://dx.doi.org/10.1103/PhysRevLett.78.998

Ferraz, K., \& Francisco, G. (1992). Mixmaster numerical behaviour and generalizations. Phys. Rev. D, 45(4), 1158-1162. http://dx.doi.org/10.1103/PhysRevD.45.1158

Hartle J., \& Hawking, S. (1983). Wave function of the universe. Phys. Rev. D, 28, 2960-2975. http://dx.doi.org/10.1103/PhysRevD.28.2960

Hawking, S. W., \& Ellis, G. F. R. (1968). The cosmic black-body radiation and the existence of singularities in our universe. Astrophys. J., 152, 25-36. http://dx.doi.org/10.1086/180171

Khalatnikov, I. M., Lifshitz, E. M., Khanin, K. M., Shchur, L. N., \& Sinai, Y. G. (1985). On the stochasticity in Relativistic Cosmology. J. Stat. Phys., 38, 97-113. http://dx.doi.org/10.1007/BF01017851

Latifi, A., Musette, M., \& Conte, R. (1994). The Bianchi IX (Mixmaster) cosmological model is not integrable. Phys. Lett. A, 194, 83-92. http://dx.doi.org/10.1016/0375-9601(94)00732-5

Lifshitz, E. M., \& Khalatnikov, I. M. (1963). Investigations in relativistic cosmology. Adv. Phys., 12, 185-249. http://dx.doi.org/10.1080/00018736300101283

Lifshitz, E. M., Lifshitz, I. M., \& Khalatnikov, I. M. (1971).Asymptotic analysis of oscillatory mode of approach to a singularity in homogeneous cosmological models. Sov. Phys. J. E. T. P., 32, 173-180.

Lin, X. F., \& Wald, R. M. (1990). Proof of the closed-universe recollapse conjecture for general Bianchi type-IX cosmologies. Phys. Rev. D, 41, 2444-2448. http://dx.doi.org/10.1103/PhysRevD.41.2444

Misner, C. W. (1969a). Mixmaster universe. Phys. Rev. Lett., 22A(20) 1071-1074. http://dx.doi.org/10.1103/PhysRevLett.22.1071 
Misner, C. W. (1969b). Quantum cosmology. Phys. Rev., 186(5), 1319-1327. http://dx.doi.org/10.1103/PhysRev.186.1319

Ryan, M. P. (1971a). Qualitative cosmology: Diagrammatic solutions for Bianchi type IX universes with expansion, rotation, and shear. Ann. Phys., 65, 505-537. http://dx.doi.org/10.1016/0003-4916(71)90178-3

Ryan, M. P. (1971b). Qualitative cosmology: Diagrammatic solutions for Bianchi type IX universes with expansion, rotation, and shear. II. The general case. Ann. Phys., 68, 541-555. http://dx.doi.org/10.1016/0003-4916(71)90135-7

Springael, J., Conte, R., \& Musette, M. (1998). On the exact solutions of the Bianchi IX cosmological model in the proper time. Regul. Chaotic Dynam., 3, 3-8. http://dx.doi.org/10.1070/rd1998v003n01ABEH000057

Wright, W. A., \& Moss, I. G. (1985). The anisotropy of the universe. Phys. Lett. B, 154(2,3), 115-119.

Zardecki, A. (1983). Modeling in chaotic relativity. Phys. Rev. D, 28, 1235-1242. http://dx.doi.org/10.1103/PhysRevD.28.1235

\section{Copyrights}

Copyright for this article is retained by the author(s), with first publication rights granted to the journal.

This is an open-access article distributed under the terms and conditions of the Creative Commons Attribution license (http://creativecommons.org/licenses/by/3.0/). 